Proyecciones Journal of Mathematics

Vol. 40, No 5, pp. 1071-1096, October 2021.

Universidad Católica del Norte

Antofagasta - Chile

\title{
Infinitely many solutions for anisotropic elliptic equations with variable exponent
}

\author{
Abdelrachid El Amrouss \\ University Mohamed I, Morocco \\ and \\ Ali El Mahraoui \\ University Mohamed I, Morocco \\ Received: December 2019. Accepted : February 2021
}

\begin{abstract}
In this article, we study the existence and multiplicity of solutions for a class of anisotropic elliptic equations

$(P)\left\{\begin{array}{l}-\sum_{i=1}^{N} \partial_{x_{i}} a_{i}\left(x, \partial_{x_{i}} u\right)+b(x)|u|^{P_{+}^{+}-2} u=\lambda(x) f(x, u)+\mu(x) g(x, u) \text { in } \Omega, \\ u=0 \quad \text { on } \quad \partial \Omega .\end{array}\right.$

First we establisch that anisotropic space is separable and by using the Fountain theorem, and dual Fountain theorem we prove, under suitable conditions, that the problem $(P)$ admits two sequences of weak solutions.
\end{abstract}

Keywords: Quasilinear elliptic equations, variable exponent Lebesgue space, anisotropic space, Fountain theorem, dual Fountain theorem.

AMS Subject Classifications: 35J25, 35J62, 35D30, 46E35, 35J20. 


\section{Introduction}

Let $\Omega \subset \mathbf{R}^{N}(N \geq 3)$ be a bounded domain with smooth boundary. In this paper we will study the multiplicity of weak solutions of the anisotropic problem:

$(P)\left\{\begin{array}{l}-\sum_{i=1}^{N} \partial_{x_{i}} a_{i}\left(x, \partial_{x_{i}} u\right)+b(x)|u|^{P_{+}^{+}-2} u=\lambda(x) f(x, u)+\mu(x) g(x, u) \text { in } \Omega, \\ u=0 \quad \text { on } \quad \partial \Omega,\end{array}\right.$

where $\lambda \not \equiv 0$ and $\mu \not \equiv 0, \quad b \in L^{\infty}(\Omega), f, g: \Omega \times \mathbf{R} \rightarrow \mathbf{R}$, and $a_{i}$ : $\Omega \times \mathbf{R} \rightarrow \mathbf{R}$ are Carathéodory functions fulfilling some natural hypotheses. The anisotropic differential operator $\sum_{i=1}^{N} \partial_{x_{i}} a_{i}\left(x, \partial_{x_{i}} u\right)$ is a $\vec{p}($.$) -Laplace$ type operator, where $\vec{p}(x)=\left(p_{1}(x), p_{2}(x), \ldots, p_{N}(x)\right)$ and $P_{+}^{+}=\max _{i \in\{1,2, \ldots, N\}} \sup _{x \in \Omega} p_{i}(x)$ for $i=1, \ldots, N$, we assume that $p_{i}$ is a continuous function on $\bar{\Omega}$ for all $i \in\{1,2, \ldots, N\}$. We denote by $a_{i}(x, \eta)$ the continuous derivative with respect to $\eta$ of the mapping $A_{i}: \Omega \times \mathbf{R} \rightarrow \mathbf{R}, A_{i}=A_{i}(x, \eta)$, that means $a_{i}(x, \eta)=\frac{\partial}{\partial \eta} A_{i}(x, \eta)$. We make the following assumptions on the mapping $A_{i}$ :

$\left(A_{0}\right) A_{i}(x, 0)=0$ for a.e. $x \in \Omega$.

$\left(A_{1}\right)$ There exists a positive constant $\bar{c}_{i}$ such that $a_{i}$ satisfies the growth condition

$$
\left|a_{i}(x, \eta)\right| \leq \bar{c}_{i}\left(1+|\eta|^{p_{i}(x)-1}\right),
$$

for all $x \in \Omega$ and $\eta \in \mathbf{R}$.

$\left(A_{2}\right)$ The inequalities

$$
|\eta|^{p_{i}(x)} \leq a_{i}(x, \eta) \eta \leq p_{i}(x) A_{i}(x, \eta),
$$

are verified for all $x \in \Omega$ and $\eta \in \mathbf{R}$.

$\left(A_{3}\right)$ assume that $a_{i}$ is strictly monotone, that is,

$$
\left(a_{i}(x, \eta)-a_{i}(x, \xi)\right)(\eta-\xi)>0,
$$

for all $x \in \Omega$ and $\eta, \xi \in \mathbf{R}$, with $\eta \neq \xi$.

$\left(A_{4}\right) A_{i}(x,-\eta)=A_{i}(x, \eta)$ for all $x \in \Omega, \eta \in \mathbf{R}$. 


\section{Examples}

1) If we take $a_{i}(x, \eta)=|\eta|^{p_{i}(x)-2} \eta$ for all $i \in\{1, \ldots, N\}$, we have $A_{i}(x, \eta)=$ $\frac{1}{p_{i}(x)}|\eta|^{p_{i}(x)}$ for all $i \in\{1, \ldots, N\}$. Obviously, $\left(A_{0}\right)-\left(A_{4}\right)$ are verified, and we obtain the $\vec{p}(x)$-Laplace operator

$$
\triangle_{\vec{p}(x)}(u)=\sum_{i=1}^{N} \partial_{x_{i}}\left(\left|\partial_{x_{i}} u\right|^{p_{i}(x)-2} \partial_{x_{i}} u\right) .
$$

2) If we take $a_{i}(x, \eta)=\left(1+\eta^{2}\right)^{\frac{p_{i}(x)-2}{2}} \eta$ for all $i \in\{1, \ldots, N\}$, we have $A_{i}(x, \eta)=\frac{1}{p_{i}(x)}\left[\left(1+|\eta|^{2}\right)^{\frac{p_{i}(x)}{2}}-1\right]$ for all $i \in\{1, \ldots, N\}$, then $\left(A_{0}\right)-\left(A_{4}\right)$ are verified, and we find the anisotropic variable exponent mean curvature operator

$$
\left.\sum_{i=1}^{N} \partial_{x_{i}}\left(1+\left|\partial_{x_{i}} u\right|^{2}\right)^{\frac{p_{i}(x)-2}{2}} \partial_{x_{i}} u\right)
$$

Anisotropic elliptic equations has been received a rising interest, in the recent years. This is due to the some nonhomogeneous materials which could not be treated on the classical Sobolev space. For example we can point out to the electro-rheological fluids or to the thermo-rheological fluids (see for example [17], [19], [23]), image restoration [22], and contact mechanics [18]. We give in this work an extension of the article [1] where the authors considered the following problem

$$
(S)\left\{\begin{array}{l}
-\sum_{i=1}^{N} \partial_{x_{i}} a_{i}\left(x, \partial_{x_{i}} u\right)+b(x)|u|^{P_{+}^{+}-2} u=f(x, u) \quad \text { in } \quad \Omega, \\
u=0 \quad \text { on } \quad \partial \Omega
\end{array}\right.
$$

and they considered $f(x, u)=\lambda|u|^{q(x)-2} u+\mu|u|^{\gamma(x)-2} u$, where $\lambda$ and $\mu$ are constant, and they established the existence of two unbounded sequences of weak solutions, their proof is based on Fountain theorem [20].

This kind of equation are treated in several works by many authors, we refer here to the articles ( [3], [6], [15], [16]). In [16], the authors considered the problem $(S)$, without $b(x)|u|^{P_{+}^{+}-2} u$, where $f(x, u)=\lambda|u|^{q(x)-2} u$, and established the existence of a continuous spectrum in several distinct situations. But in [15], the authors took the same problem with $\lambda$ depends on the variable $x$, using the mountain-pass theorem of Ambrosetti and Rabinowitz [3] and the Ekelandes variational principle, they proved that under suitable conditions, problem $(S)$ has two nontrivial weak solutions. In [6], 
M. M. Boureanu, P. Pucci, and V. Rădulescu proved that problem $(S)$ has a sequence of weak solutions by means of the symmetric mountain-pass theorem .

Given $\Omega \subset \mathbf{R}^{N}$, we set

$$
C_{+}(\bar{\Omega})=\left\{h \in C(\bar{\Omega}) \mid \min _{x \in \bar{\Omega}} h(x)>1\right\} .
$$

For any $h \in C_{+}(\bar{\Omega})$, we define

$$
h^{+}=\sup _{x \in \bar{\Omega}} h(x) \quad \text { and } \quad h^{-}=\inf _{x \in \bar{\Omega}} h(x) .
$$

Let $p \in C_{+}(\bar{\Omega})$, then $L^{p(x)}(\Omega)$ is called variable exponent Lebesgue space which is defined as follow

$$
\begin{aligned}
& L^{p(x)}(\Omega)=\{u: u \text { is a measurable real-valued function such that } \\
& \left.\qquad \int_{\Omega}|u(x)|^{p(x)} d x<\infty\right\},
\end{aligned}
$$

endowed with the Luxemburg norm

$$
|u|_{L^{p(x)}(\Omega)}=|u|_{p(x)}=\inf \left\{\mu>0: \int_{\Omega}\left|\frac{u(x)}{\mu}\right|^{p(x)} d x \leq 1\right\}
$$

is a separable and reflexive Banach space (see [21]).

In order to study the problem $(P)$ we have to introduce the vectors $\vec{P}_{+}, \vec{P}_{-} \in \mathbf{R}^{N}, p_{+}(x)$ and $p_{-}(x)$ which are defined in the following way

$$
\begin{gathered}
\vec{P}_{+}=\left(p_{1}^{+}, p_{2}^{+}, \ldots, p_{N}^{+}\right), \vec{P}_{-}=\left(p_{1}^{-}, p_{2}^{-}, \ldots, p_{N}^{-}\right), \\
p_{+}(x)=\max \left\{p_{1}(x), \ldots, p_{2}(x)\right\}, p_{-}(x)=\min \left\{p_{1}(x), \ldots, p_{2}(x)\right\},
\end{gathered}
$$

and the positive real numbers $P_{+}^{+}, P_{-}^{+}, P_{-}^{-}$as the following

$$
P_{+}^{+}=\max \left\{p_{1}^{+}, \ldots, p_{N}^{+}\right\}, P_{-}^{+}=\max \left\{p_{1}^{-}, \ldots, p_{N}^{-}\right\}, P_{-}^{-}=\min \left\{p_{1}^{-}, \ldots, p_{N}^{-}\right\} .
$$

We say that $p$ is logarithmic Hölder continuous if

(1.1) $|p(x)-p(y)| \leq-\frac{M}{\log (|x-y|)} \forall x, y \in \Omega$ such that $|x-y| \leq 1 / 2$. 
The variable exponent Sobolev space $W^{1, p(x)}(\Omega)$ is defined by

$$
W^{1, p(x)}(\Omega)=\left\{u \in L^{p(x)}(\Omega): \nabla u \in\left[L^{p(x)}(\Omega)\right]^{N}\right\} .
$$

For all $u \in W^{1, p(x)}(\Omega)$, we have $\|u\|_{1, p(x)}=|u|_{p(x)}+|\nabla u|_{p(x)}$. If $p$ satisfies (1.1), the space $W_{0}^{1, p(x)}(\Omega)$ is the closure of $C_{0}^{\infty}(\Omega)$ in $W^{1, p(x)}(\Omega)$ under the norm $\|u\|_{1, p(x)}$. For $u \in W_{0}^{1, p(x)}(\Omega)$, we can define an equivalent norm $\|u\|_{p(x)}=|\nabla u|_{p(x)}$.

Now, we introduce a natural generalization of the function space $W^{1, p(x)}(\Omega)$, which is called anisotropic variable exponent Sobolev space $W^{1, \vec{p}(x)}(\Omega)$ and we have

$$
W^{1, \vec{p}(x)}(\Omega)=\left\{u \in L^{p_{+}(x)}(\Omega): \partial_{x_{i}} u \in L^{p_{i}(x)}(\Omega), \forall i \in\{1, \ldots, N\}\right\} .
$$

If $\vec{p}: \bar{\Omega} \rightarrow \mathbf{R}^{N} ; \quad \vec{p}(x)=\left(p_{1}(x), p_{2}(x), \ldots, p_{N}(x)\right)$, and for each $i \in$ $\{1,2, \ldots, N\}$, we have $p_{i} \in C_{+}(\bar{\Omega})$, and satisfy (1.1), the anisotropic variable exponent Sobolev space $W_{0}^{1, \vec{p}(x)}(\Omega)$ is defined as the closure of $C_{0}^{\infty}(\Omega)$ under the norm

$$
\|u\|=\|u\|_{\vec{p}(.)}=\sum_{i=1}^{N}\left|\partial_{x_{i}} u\right|_{p_{i}(.)},
$$

and ites a reflexive Banach space $($ see $[9,16])$.

Throughout this paper, we assume that

$$
\sum_{i=1}^{N} \frac{1}{p_{i}^{-}}>1
$$

Define $P_{-}^{*}, P_{-, \infty} \in \mathbf{R}^{+}$by

$$
P_{-}^{*}=\frac{N}{\sum_{i=1}^{N} \frac{1}{p_{i}^{-}}-1}, P_{-, \infty}=\max \left\{P_{-}^{+}, P_{-}^{*}\right\} .
$$

In this paper, we have $P_{+}^{+}<P_{-, \infty}=\max \left\{P_{-}^{+}, P_{-}^{*}\right\}=P_{-}^{*}$.

From now on, we put $X=W_{0}^{1, \vec{p}(x)}(\Omega)$.

Let us define $F(x, t)=\int_{0}^{t} f(x, s) d s$ and $G(x, t)=\int_{0}^{t} g(x, s) d s$, and we assume that the following hypotheses are fulfilled:

$\left(f_{0}\right)|f(x, t)| \leq c|t|^{\alpha(x)-1}$, for all $(x, t) \in \Omega \times \mathbf{R}$ where $c>0$ is a constant, $\alpha \in C_{+}(\Omega)$ such that $\alpha^{+}=\sup _{\bar{\Omega}} \alpha(x)<P_{-}^{-}<P_{+}^{+} \leq s(x)<$ 
$P_{-, \infty}, \forall x \in \Omega$ and $\lambda \in L^{\frac{s(x)}{s(x)-\alpha(x)}}(\Omega)$, with $s \in C_{+}(\bar{\Omega})$ and $\alpha(x)+1 \leq$ $s(x)<P_{-, \infty}, \forall x \in \Omega$.

$\left(f_{1}\right) F(x, t) \geq h_{0}(x) t^{\alpha_{0}(x)}$ when $t \rightarrow 0^{+}$, and $\alpha_{0} \in C_{+}(\bar{\Omega})$ with $\alpha_{0}^{+}<$ $1, h_{0}(x) \geq 0 \forall x \in \Omega, \not \equiv 0$, and also $h_{0} \in C(\Omega, \mathbf{R})$.

$\left(f_{2}\right) f(x,-t)=-f(x, t) \forall x \in \Omega, t \in \mathbf{R}$.

$\left(g_{0}\right)|g(x, t)|<d|t|^{\gamma(x)-1}$, for all $(x, t) \in \Omega \times \mathbf{R}$ where $d>0$ is a constant, and $\gamma \in C_{+}(\bar{\Omega})$ satisfying $P_{+}^{+}<\gamma^{-}<\gamma^{+}<P_{-, \infty}$, and $\mu \in$ $L^{\frac{r(x)}{r(x)-\gamma(x)}}(\Omega)$, with $r \in C_{+}(\bar{\Omega})$, and $\gamma(x)+1 \leq r(x)<P_{-, \infty} \forall x \in \Omega$.

$\left(g_{1}\right)$ There exists a constant $\theta>P_{+}^{+}$such that $0<\theta G(x, t) \leq t g(x, t)$, for a.e. $x \in \Omega, t \in \mathbf{R}$.

$\left(g_{1}^{\prime}\right)$ There exist constants $\beta>\gamma(x)>\theta>P_{+}^{+}$such that $0<\theta G(x, t) \leq$ $\operatorname{tg}(x, t) \leq \beta G(x, t)$, for a.e. $x \in \Omega, t \in \mathbf{R}$.

$\left(g_{2}\right) g(x,-t)=-g(x, t) \quad$ for all $x \in \Omega, t \in \mathbf{R}$.

(B) $b \in L^{\infty}(\Omega)$ and there exists $b_{0}>0$ such that $b(x) \geq b_{0}$ for all $x \in \Omega$.

The main result of this paper is as follows.

Theorem 1.1. Assume $\left(A_{0}\right)-\left(A_{4}\right),\left(f_{0}\right)-\left(f_{2}\right)$ and $\left(g_{0}\right)-\left(g_{2}\right)$ and $(B)$, then

1. If $\mu(x)>0$ a.e. $x \in \Omega$ and $\lambda(x) \in \mathbf{R}$, problem $(P)$ has a sequence of weak solutions $\left( \pm u_{k}\right)$ such that $\Phi\left( \pm u_{k}\right) \rightarrow+\infty$ as $k \rightarrow+\infty$.

2. If $\lambda(x)>0, \mu(x)>0$ a.e. $x \in \Omega$, problem $(P)$ has a sequence of weak solutions $\left( \pm v_{k}\right)$ such that $\Phi\left( \pm v_{k}\right) \rightarrow 0$ as $k \rightarrow+\infty$.

3. If $\lambda(x)>0$ a.e. $x \in \Omega$ and $\mu(x)$ keeps a constant sign in $\mathbf{R}$, and $g$ satisfies $\left(g_{1}^{\prime}\right)$ instead of $\left(g_{1}\right)$, problem $(P)$ has a sequence of weak solutions $\left( \pm v_{k}\right)$ such that $\Phi\left( \pm v_{k}\right) \rightarrow 0$ as $k \rightarrow+\infty$. 
Remark 1.1. 1. Among the difficulties we have raised in this pape is to prove that anisotropic space is separable. To my knowledge, I believe that the demonstration of this result is not found in the literature.

2. For $f(x, u)=|u|^{\alpha(x)-2} u, g(x, u)=|u|^{\gamma(x)-2} u, \quad \lambda(x)=\lambda \in \mathbf{R}$ and $\mu(x)=\mu$ for all $x \in \Omega$ with $\alpha(x)<P_{-}^{-}<P_{+}^{+}<P_{-, \infty}, \forall x \in \Omega$, and $P_{+}^{+}<\gamma^{-}<\gamma^{+}<P_{-, \infty}$, we obtain the result in the article [1].

This paper is divided into two sections. In the first section we will give some known results, in the second we will give the proof of our main results.

\section{Preliminaries}

We recall some important definitions and properties of the Lebesgue and Sobolev spaces with variable exponent $L^{p(x)}(\Omega)$ and $W_{0}^{1, \vec{p}(x)}(\Omega)$, where $\Omega$ is a bounded domain in $\mathbf{R}^{N}$.

Proposition 2.1. (see $[8,13,12])$

1. The space $\left(L^{p(x)}(\Omega),|u|_{p(x)}\right)$ is a separable, uniformly convex Banach space and its dual space is $L^{q(x)}(\Omega)$, where $\frac{1}{p(x)}+\frac{1}{q(x)}=1$. For any $u \in L^{p(x)}(\Omega)$ and $v \in L^{q(x)}(\Omega)$, we have

$$
\left|\int_{\Omega} u v d x\right| \leq\left(\frac{1}{p^{-}}+\frac{1}{q^{-}}\right)|u|_{p(x)}|v|_{q(x)} \leq 2|u|_{p(x)}|v|_{q(x)} .
$$

2. If $p_{1}(x), p_{2}(x) \in C_{+}(\bar{\Omega}), p_{1}(x) \leq p_{2}(x), \forall x \in \bar{\Omega}$, then $L^{p_{2}(x)}(\Omega) \hookrightarrow$ $L^{p_{1}(x)}(\Omega)$ and the embedding is continuous.

Remark: If $\frac{1}{p(x)}+\frac{1}{q(x)}+\frac{1}{r(x)}=1$, then for any $u \in L^{p(x)}(\Omega), v \in$ $L^{q(x)}(\Omega), w \in L^{r(x)}(\Omega)$, we have

$\left|\int_{\Omega} u v w d x\right| \leq\left(\frac{1}{p^{-}}+\frac{1}{q^{-}}+\frac{1}{r^{-}}\right)|u|_{p(x)}|v|_{q(x)}|w|_{r(x)} \leq 3|u|_{p(x)}|v|_{q(x)}|w|_{r(x)}$. 
Lemma 2.1. (see[2]) Let $q, s \in C_{+}(\bar{\Omega})$ with $q(x) \leq s(x)$ for all $x \in \bar{\Omega}$, and $u \in L^{s(x)}(\Omega)$, then $|u|^{q(x)} \in L^{\frac{s(x)}{q(x)}}(\Omega)$ and

$$
\left.\left.|| u\right|^{q(x)}\right|_{\frac{s(x)}{q(x)}} \leq|u|_{s(x)}^{q^{+}}+|u|_{s(x)}^{q^{-}}
$$

or there exists a number $\tilde{q} \in\left[q^{-}, q^{+}\right]$such that

$$
\left.\left.|| u\right|^{q(x)}\right|_{\frac{s(x)}{q(x)}}=|u|_{s(x)}^{\tilde{q}} .
$$

Proposition 2.2. (see[11]) Denote $\rho_{p(x)}(u)=\int_{\Omega}|u(x)|^{p(x)} d x$. Then for $u \in L^{p(x)}(\Omega),\left(u_{n}\right) \subset L^{p(x)}(\Omega)$ we have

1. $|u|_{p(x)}<1(=1 ;>1) \Leftrightarrow \rho_{p(x)}(u)<1(=1 ;>1)$,

2. $|u|_{p(x)}>1 \Rightarrow|u|_{p(x)}^{p^{-}} \leq \rho_{p(x)}(u) \leq|u|_{p(x)}^{p^{+}}$,

3. $|u|_{p(x)}<1 \Rightarrow|u|_{p(x)}^{p^{+}} \leq \rho_{p(x)}(u) \leq|u|_{p(x)}^{p^{-}}$,

4. $|u|_{p(x)} \rightarrow 0(\rightarrow \infty) \Leftrightarrow \rho_{p(x)}(u) \rightarrow 0(\rightarrow \infty)$,

5. $\lim _{n \rightarrow \infty}\left|u_{n}-u\right|_{p(x)}=0 \Leftrightarrow \lim _{n \rightarrow \infty} \rho_{p(x)}\left(u_{n}-u\right)=0$.

We recall now some results which concerning the embedding theorem.

Proposition 2.3. (see[16]) Suppose that $\Omega \subset \mathbf{R}^{N}(N>3)$ is a bounded domain with smooth boundary and relation (1.2) is fulfilled.

1. For any $q \in C(\bar{\Omega})$ verifying

$$
1<q(x)<P_{-, \infty} \forall x \in \bar{\Omega},
$$

the embedding

$$
W_{0}^{1, \vec{p}(x)}(\Omega) \hookrightarrow L^{q(x)}(\Omega)
$$

is continuous and compact.

2. Assume that $P_{-}^{-}>N$, then the embedding

$$
W_{0}^{1, \vec{p}(x)}(\Omega) \hookrightarrow C(\bar{\Omega})
$$

is continuous and compact. 
Under the conditions $\left(A_{i}\right), i=0,1,2,3$, we have the proposition below which is useful.

Proposition 2.4. (cf.[14, 5]) Let

$$
\mathcal{A}_{i}(u)=\int_{\Omega} A_{i}\left(x, \partial_{x_{i}} u\right) d x
$$

For $i \in\{1,2, \ldots, N\}$, we have:

- $\mathcal{A}_{i}$ is well defined on $X$,

- the functional $\mathcal{A}_{i} \in C^{1}(X, \mathbf{R})$ and

$$
\left\langle\mathcal{A}_{i}^{\prime}(u), \varphi\right\rangle=\int_{\Omega} a_{i}\left(x, \partial_{x_{i}} u\right) \partial_{x_{i}} \varphi d x,
$$

for all $u, \varphi \in X$.

- $\mathcal{A}_{i}$ is weakly lower semi-continuous.

- Let

$$
\mathcal{A}(u)=\int_{\Omega} \sum_{i=1}^{N} A_{i}\left(x, \partial_{x_{i}} u\right) d x
$$

then $\mathcal{A}^{\prime}$ is an operator of type $\left(S_{+}\right)(c f .[5])$. tion.

The following lemma plays an important role to define the weak solu-

Lemma 2.2. Let $B(u)=\int_{\Omega}[\lambda(x) F(x, u)+\mu(x) G(x, u)] d x$. Suppose that $\left(f_{0}\right)$ and $\left(g_{0}\right)$ are verified, then we have

1. $B$ is well defined and $B \in C^{1}(X, \mathbf{R})$.

2. $B, B^{\prime}$ are weakly-strongly continuous.

Proof. 1.) As $f$ and $g$ satisfy $\left(f_{0}\right)$ and $\left(g_{0}\right)$, using lemma 2.1 and proposition 2.3, we have 


$$
\begin{aligned}
\int_{\Omega}|\lambda(x) F(x, u)+\mu(x) G(x, u)| d x & \leq \int_{\Omega}|\lambda(x)||F(x, u)| d x+\int_{\Omega}|\mu(x) \| G(x, u)| d x \\
& \leq 2 \frac{c}{\alpha^{-}}|\lambda|_{\frac{s(x)}{s(x)-\alpha(x)}\left|u^{\alpha(x)}\right|_{\frac{s(x)}{\alpha(x)}}} \\
& +2 \frac{d}{\gamma^{-}}|\mu|_{\frac{r(x)}{r(x)-\gamma(x)}\left|u^{\gamma(x)}\right|_{\frac{s(x)}{\gamma(x)}}} \\
& \leq 2 c_{1} \frac{c}{\alpha^{-}}|\lambda|_{\frac{s(x)}{s(x)-\alpha(x)}}\|u\|^{\tilde{\alpha}}+2 d_{1} \frac{d}{\gamma^{-}}|\mu|_{\frac{r(x)}{r(x)-\gamma(x)}}\|u\| \|^{\tilde{\gamma}} .
\end{aligned}
$$

The last expression is obtained by the compact embeddings $X \hookrightarrow$ $L^{s(x)}(\Omega)$ and $X \hookrightarrow L^{r(x)}(\Omega)$, which means that there exist constants $c_{1}>0$ and $d_{1}>0$ such that $|u|_{s(x)} \leq c_{1}\|u\|$, and $|u|_{r(x)} \leq d_{1}\|u\|$, so $B$ is well defined. Let us prove that $B \in C^{1}(X, \mathbf{R})$. We show first that $B$ is Gâteaux differentiable. Using the Mean-value theorem, we have

$$
\begin{aligned}
D B(u, \varphi) & =\lim _{t \rightarrow 0} \frac{B(u+t \varphi)-B(u)}{t} \\
& =\lim _{t \rightarrow 0} \int_{\Omega} \frac{\lambda(x) F(x, u+t \varphi)-\lambda(x) F(x, u)+\mu(x) G(x, u+t \varphi)-\mu(x) G(x, u)}{t} d x \\
& =\lim _{t \rightarrow 0} \int_{\Omega}[\lambda(x) f(x, u+t \theta \varphi)+\mu(x) g(x, u+t \theta \varphi)] \varphi(x) d x,
\end{aligned}
$$

where $0 \leq \theta \leq 1$. From $\left(f_{0}\right),\left(g_{0}\right)$, Young's inequality, and the convexity of the function $h(a)=|a|^{p}$ with $p \geq 1$, for $|t| \leq 1$, we have

$[\lambda(x) f(x, u+t \theta \varphi)+\mu(x) g(x, u+t \theta \varphi)] \varphi(x)$

$\leq c|\lambda(x)||u+t \theta \varphi|^{\alpha(x)-1}|\varphi(x)|+d|\mu(x)||u+t \theta \varphi|^{\gamma(x)-1}|\varphi(x)|$,

$\leq \frac{c(s(x)-\alpha(x))}{s(x)}|\lambda(x)|^{\frac{s(x)}{s(x)-\alpha(x)}}+\frac{c(\alpha(x)-1)}{s(x)}\left[|u+t \theta \varphi|^{\alpha(x)-1}\right]^{\frac{s(x)-1}{\alpha(x)}}$

$+c|\varphi(x)|^{s(x)}+\frac{d(r(x)-\gamma(x))}{r(x)}|\mu(x)|^{\frac{r(x)}{r(x)-\gamma(x)}}$

$+\frac{d(\gamma(x)-1)}{r(x)}\left[|u+t \theta \varphi|^{\gamma(x)-1}\right]^{\frac{r(x)}{\gamma(x)-1}}+d|\varphi(x)|^{r(x)}$,

$\leq \frac{c(s(x)-\alpha(x))}{s(x)}|\lambda(x)|^{\frac{s(x)}{s(x)-\alpha(x)}}+\frac{c(\alpha(x)-1)}{s(x)} 2^{s(x)-1}\left[|u|^{s(x)}+|\varphi|^{s(x)}\right]$

$+c|\varphi(x)|^{s(x)}+\frac{d(r(x)-\gamma(x))}{r(x)}|\mu(x)|^{\frac{r(x)}{r(x)-\gamma(x)}}$

$+\frac{d(\gamma(x)-1)}{r(x)} 2^{r(x)-1}\left[|u|^{r(x)}+|\varphi|^{r(x)}\right]+d|\varphi(x)|^{r(x)}$. 
The last right expression is independent on $t$ and it is in $L^{1}(\Omega)$, then by the Lebesgue dominated convergence theorem, we have

$$
D B(u, \varphi)=\int_{\Omega}[\lambda(x) f(x, u)+\mu(x) g(x, u)] \varphi(x) d x .
$$

So using the fact that $N_{f}: L^{s(x)}(\Omega) \rightarrow L^{\frac{s(x)}{\alpha(x)-1}}(\Omega) ; u \mapsto f(x, u)$ and $N_{g}$ : $L^{r(x)}(\Omega) \rightarrow L^{\frac{r(x)}{\gamma(x)-1}}(\Omega) ; u \mapsto g(x, u)$ are continuous bounded operators. Then, by $\left(f_{0}\right),\left(g_{0}\right)$, and Proposition 2.1, we obtain

$$
\begin{aligned}
D B(u, \varphi) & =\int_{\Omega}[\lambda(x) f(x, u)+\mu(x) g(x, u)] \varphi(x) d x \\
& \leq \int_{\Omega} c|\lambda(x)||f(x, u)||\varphi(x)| d x+\int_{\Omega} d|\mu(x)||g(x, u)||\varphi(x)| d x \\
& \leq 3 c|\lambda|_{\frac{s(x)}{s(x)-\alpha(x)}}|f(x, u)|_{\frac{s(x)}{\alpha(x)-1}}|\varphi(x)|_{s(x)} \\
& +3 d|\mu|_{\frac{r(x)}{r(x)-\gamma(x)}}|g(x, u)|_{\frac{r(x)}{\gamma(x)-1}}|\varphi(x)|_{r(x)} .
\end{aligned}
$$

So $D B(u, \varphi)$, as a function of $\varphi$, is a continuous linear functional on $X$, then it is the Gâteaux differential of $B$. Let's prove that it's continuous, then for $u, v, \varphi \in X$, from (2.2), we get

$$
\begin{aligned}
|\langle D B(u)-D B(v), \varphi\rangle| & \leq 3|\lambda|_{\frac{s(x)}{s(x)-\alpha(x)}}|f(x, u)-f(x, v)|_{\frac{s(x)}{\alpha(x)-1}}|\varphi|_{s(x)} \\
& +3|\mu|_{\frac{r(x)}{r(x)-\gamma(x)}}|g(x, u)-g(x, v)|_{\frac{r(x)}{\gamma(x)-1}}|\varphi|_{r(x)} \\
& \leq K_{1}|f(x, u)-f(x, v)|_{\frac{s(x)}{\alpha(x)-1}}\|\varphi\|+K_{2} \mid g(x, u) \\
& -\left.g(x, v)\right|_{\frac{r(x)}{\gamma(x)-1}}\|\varphi\|,
\end{aligned}
$$

where $K_{1}>0$ and $K_{2}>0$ are constants. Then,

$$
\|D B(u)-D B(v)\|_{X^{*}} \leq K_{1}|f(x, u)-f(x, v)|_{\frac{s(x)}{\alpha(x)-1}}+K_{2}|g(x, u)-g(x, v)|_{\frac{r(x)}{\gamma(x)-1}} .
$$

Thus, $D B(u)$ is continuous, so $B$ is Frèchet differentiable and $B \in C^{1}(X, \mathbf{R})$ with

$$
\left\langle B^{\prime}(u), \varphi\right\rangle=\int_{\Omega}[\lambda(x) f(x, u)+\mu(x) g(x, u)] \varphi(x) d x .
$$


2.) Suppose by contradiction that there exists a sequence $\left(u_{n}\right) \subset X$ such that $u_{n} \rightarrow u$ and $B\left(u_{n}\right) B(u)$, then there exists $\varepsilon_{0}$ and subsequence still denoted $\left(u_{n}\right)$ such that:

$$
0<\varepsilon_{0} \leq\left|B\left(u_{n}\right)-B(u)\right| .
$$

For $0<\theta_{n}<1$, and by finite increment theorem we have

$$
0<\varepsilon_{0} \leq\left|\left\langle B^{\prime}\left(u_{n}+\theta_{n}\left(u_{n}-u\right)\right), u_{n}-u\right\rangle\right| .
$$

Put $w_{n}=u_{n}+\theta_{n}\left(u_{n}-u\right)$. As $B^{\prime}(u)(w)=\int_{\Omega}[\lambda(x) f(x, u) w+\mu(x) g(x, u) w] d x$, using (2.1), proposition $2.3,\left(f_{0}\right)$ and $\left(g_{0}\right)$ we obtain

$$
\begin{aligned}
\left|\left\langle B^{\prime}\left(w_{n}\right),\left(u_{n}-u\right)\right\rangle\right| & =\int_{\Omega}\left|\lambda(x) f\left(x, w_{n}\right)\left(u_{n}-u\right)+\mu(x) g\left(x, w_{n}\right)\left(u_{n}-u\right)\right| d x \\
& \leq \int_{\Omega}\left\{|\lambda(x)|\left|u_{n}-u\right|\left|f\left(x, w_{n}\right)\right|+|\mu(x)|\left|u_{n}-u \| g\left(x, w_{n}\right)\right|\right\} d x \\
& \leq \int_{\Omega} c|\lambda(x)|\left|w_{n}\right|^{\alpha(x)-1}\left|u_{n}-u\right| d x|| \\
& +\int_{\Omega} d|\mu(x)|\left|w_{n}\right|^{\gamma(x)-1}\left|u_{n}-u\right| d x \\
& \leq\left.\left. 3 c|\lambda(x)|_{\frac{s(x)}{s(x)-\alpha(x)}}|| w_{n}\right|^{\alpha(x)-1}\right|_{\frac{s(x)}{\alpha(x)-1}}\left|u_{n}-u\right|_{s(x)} \\
& +\left.\left.3 d|\mu(x)|_{\frac{r(x)}{r(x)-\gamma(x)}}|| w_{n}\right|^{\gamma(x)-1}\right|_{\frac{r(x)}{\gamma(x)-1}}\left|u_{n}-u\right|_{r(x)} .
\end{aligned}
$$

Since $\lim _{n \rightarrow+\infty}\left|w_{n}\right|_{s(x)} \neq \infty$ and $\lim _{n \rightarrow+\infty}\left|w_{n}\right|_{r(x)} \neq \infty$, then by the proposition 2.2 , we deduce that

$$
\left.\left.\lim _{n \rightarrow+\infty}|| w_{n}\right|^{\alpha(x)-1}\right|_{\frac{s(x)}{\alpha(x)-1}} \neq \infty \text { and }\left.\left.\lim _{n \rightarrow+\infty}|| w_{n}\right|^{\gamma(x)-1}\right|_{\frac{r(x)}{\gamma(x)-1}} \neq \infty .
$$

So, as the embeddings $X \hookrightarrow L^{s(x)}(\Omega)$ and $X \hookrightarrow L^{r(x}(\Omega)$ are compact, then the last expression on the right goes to 0 as $n \rightarrow+\infty$. Finally, $B$ is weakly-strongly continuous.

Let's prove that $B^{\prime}$ is also weakly-strongly continuous. We know that

$$
\left\langle B^{\prime}(u), v\right\rangle=\int_{\Omega} \lambda(x) f(x, u) v d x+\int_{\Omega} \mu(x) g(x, u) v d x,
$$

where $v \in X$. For $u_{n} \rightarrow u$, then $\left(u_{n}\right)$ is bounded, using relation (2.1), we have 


$$
\begin{aligned}
\left|\left\langle B^{\prime}\left(u_{n}\right)-B^{\prime}(u), v\right\rangle\right| & \leq \int_{\Omega}|\lambda(x)|\left|\left(f\left(x, u_{n}\right)-f(x, u)\right) v\right| d x \\
& +\int_{\Omega}|\mu(x)|\left|\left(g\left(x, u_{n}\right)-g(x, u)\right) v\right| d x \\
& \leq 3|\lambda|_{\frac{s(x)}{s(x)-\alpha(x)}}\left|f\left(x, u_{n}\right)-f(x, u)\right|_{\frac{s(x)}{\alpha(x)-1}}|v|_{s(x)} \\
& +3|\mu|_{\frac{r(x)}{r(x)-\gamma(x)}}\left|g\left(x, u_{n}\right)-g(x, u)\right|_{\frac{r(x)}{\gamma(x)-1}}|v|_{r(x)} .
\end{aligned}
$$

The compact embedding $X \hookrightarrow L^{s(x)}(\Omega)$ (respectively $X \hookrightarrow L^{r(x)}(\Omega)$ ) guarantees the existence of subsequence $\left(u_{n}\right)$ which converges to $u$ in $L^{s(x)}(\Omega)$ (respectively $L^{r(x)}(\Omega)$ ). So, using the continuity of $N_{f}$ and $N_{g}$, we deduce easily that $B^{\prime}$ is weakly-strongly continuous.

Let us define now the functional $\Phi$ associated with the problem $(P): \Phi$ : $X \longrightarrow \mathbf{R}$

$$
\Phi(u)=\int_{\Omega}\left[\sum_{i=1}^{N} A_{i}\left(x, \partial_{x_{i}} u\right)+\frac{b(x)}{P_{+}^{+}}|u|^{P_{+}^{+}}-\lambda(x) F(x, u)-\mu(x) G(x, u)\right] d x .
$$

Under assumptions $\left(A_{0}\right),\left(A_{1}\right),\left(f_{0}\right),(B)$ and $\left(g_{0}\right)$, we have $\Phi$ is well defined on $X$ and $\Phi \in C^{1}(X, \mathbf{R})$, moreover the conditions $\left(A_{4}\right),\left(f_{2}\right)$ and $\left(g_{2}\right)$ imply that $\Phi$ is even. So we can define a weak solution as below.

Definition 2.1. A function $u$ is a weak solution of the problem $(P)$ if and only if

$\int_{\Omega}\left[\sum_{i=1}^{N} a_{i}\left(x, \partial_{x_{i}} u\right) \partial_{x_{i}} \varphi+b(x)|u|^{P_{+}^{+}-2} u \varphi-\lambda(x) f(x, u) \varphi-\mu(x) g(x, u) \varphi\right] d x=0$,

for all $\varphi \in X$.

\section{Existence of sequences of weak solutions}

We start with proving some results and lemmas before to deduce the proof of our main results.

Proposition 3.1. $X$ is a separable Banach space. 
Proof Let us define $Y=L^{p^{+}(x)}(\Omega) \times L^{p_{1}(x)}(\Omega) \times L^{p_{2}(x)}(\Omega) \times \ldots \times L^{p_{N}(x)}(\Omega)$, endowed with the product norm is a separable Banach space, and consider the operator $T: W^{1, \vec{p}(x)}(\Omega) \rightarrow Y$, defined by $T(u)=(u, \nabla u)$. We have $\|T(u)\|_{Y}=|u|_{p^{+}(x)}+\sum_{i=1}^{N}\left|\partial_{x_{i}} u\right|_{p_{i}(x)}=\|u\|_{W^{1, \vec{p}(x)}(\Omega)}$. Thus $T$ is an isometric isomorphism of $W^{1, \vec{p}(x)}(\Omega)$ onto a subspace $W=T\left(W^{1, \vec{p}(x)}(\Omega)\right)$ of $Y$. As $W^{1, \vec{p}(x)}(\Omega)$ is complete, then $W$ is a closed subspace of $Y$. As $Y$ is a separable banach space, we will show that $W$ is a separable space. Let us consider a sequence $\left(y_{n}\right)_{n>0}$ which is dense in $Y$. For all $n \in \mathbf{N}^{*}$, let us fix a point $w_{n} \in W$ which verifies $\left\|y_{n}-w_{n}\right\|<\inf _{w \in W}\left\|y_{n}-w\right\|+\frac{1}{n}$. For $w \in W$, for all $\varepsilon>0$ and there exists $n>\frac{3}{\varepsilon}$ such that $\left\|y_{n}-w\right\|<\frac{\varepsilon}{3}$, so by the definition of the sequence $\left(w_{n}\right)$, we have $\left\|y_{n}-w_{n}\right\|<\frac{\varepsilon}{3}+\frac{1}{n}<\frac{2}{3} \varepsilon$, therefore

$$
\left\|w_{n}-w\right\|<\left\|y_{n}-w\right\|+\left\|y_{n}-w_{n}\right\|<\varepsilon .
$$

Thus, $\left(w_{n}\right)$ is a countable dense sequence in $W$. Consequently, $W$ is a separable Banach space. Thus $W$ and $W^{1, \vec{p}(x)}(\Omega)=T^{-1}(W)$ are separable. By the definition of $X$, and the equivalence of the norms $\|\cdot\|$ and $\|\cdot\|_{W^{1, \vec{p}(x)}(\Omega)}$, we can prove easily that $X$ is a closed subspace of $W^{1, \vec{p}(x)}(\Omega)$ and then $X$ is a separable Banach space.

Lemma 3.1. (see[6]) Let $u \in X$.

1. When $\|u\|<1$, we have $\sum_{i=1}^{N} \int_{\Omega}\left|\partial_{x_{i}}(u)\right|^{p_{i}(x)} d x \geq \frac{\|u\|_{+}^{P_{+}^{+}}}{N^{P_{+}^{+}-1}}$.

2. When $\|u\|>1$, we have $\sum_{i=1}^{N} \int_{\Omega}\left|\partial_{x_{i}}(u)\right|^{p_{i}(x)} d x \geq \frac{\|u\|_{-}^{P^{-}}}{N_{-}^{P_{-}^{-}}}-N$.

Lemma 3.2. The functional $\Phi$ satisfies the Palais-Smale condition.

Proof. Let $\left\{u_{n}\right\}$ be a $(P S)$ sequence, namely, $\left|\Phi\left(u_{n}\right)\right| \leq R$, where $R>0$, and $\Phi^{\prime}\left(u_{n}\right) \rightarrow 0$, as $n \rightarrow \infty$, then when $\left\|u_{n}\right\| \geq 1$, and $\lambda(x) \in \mathbf{R}$, for all $x \in \Omega$, using $\left(f_{0}\right),\left(g_{1}\right),(B)$ and $\theta>P_{+}^{+}$, we have

$$
\begin{aligned}
1+R+\left\|u_{n}\right\| & \geq \Phi\left(u_{n}\right)-\frac{1}{\theta}\left\langle\Phi^{\prime}\left(u_{n}\right), u_{n}\right\rangle \\
& \geq \int_{\Omega}\left\{\sum_{i=1}^{N} A_{i}\left(x, \partial_{x_{i}} u_{n}\right)+\frac{b(x)}{P_{+}^{+}}\left|u_{n}\right|^{P_{+}^{+}}-\lambda(x) F\left(x, u_{n}\right)\right.
\end{aligned}
$$




$$
\begin{aligned}
& \text { - } \left.\mu(x) G\left(x, u_{n}\right)\right\} d x \\
& -\frac{1}{\theta} \int_{\Omega}\left\{\sum_{i=1}^{N} a_{i}\left(x, \partial_{x_{i}} u_{n}\right) \partial_{x_{i}} u_{n}+b(x)\left|u_{n}\right|^{P_{+}^{+}}-\lambda(x) f\left(x, u_{n}\right) u_{n}\right. \\
& \text { - } \left.\mu(x) g\left(x, u_{n}\right) u_{n}\right\} d x \\
& \geq \int_{\Omega}\left\{\sum_{i=1}^{N} A_{i}\left(x, \partial_{x_{i}} u_{n}\right)-\frac{1}{\theta} a_{i}\left(x, \partial_{x_{i}} u_{n}\right) \partial_{x_{i}} u_{n}\right\} d x \\
& +\left(\frac{1}{P_{+}^{+}}-\frac{1}{\theta}\right) \int_{\Omega} b(x)\left|u_{n}\right|^{P_{+}^{+}} d x+\int_{\Omega} \mu(x)\left(\frac{1}{\theta} g\left(x, u_{n}\right) u_{n}-G\left(x, u_{n}\right)\right) d x \\
& +\int_{\Omega} \lambda(x)\left(\frac{1}{\theta} f\left(x, u_{n}\right) u_{n}-F\left(x, u_{n}\right)\right) d x \\
& \geq \int_{\Omega}\left\{\sum_{i=1}^{N} A_{i}\left(x, \partial_{x_{i}} u_{n}\right)-\frac{1}{\theta} a_{i}\left(x, \partial_{x_{i}} u_{n}\right) \partial_{x_{i}} u_{n}\right\} d x \\
& -c\left(\frac{1}{\theta}+\frac{1}{\alpha^{-}}\right) \int_{\Omega}|\lambda(x)|\left|u_{n}\right|^{\alpha(x)} d x .
\end{aligned}
$$

From $\left(A_{2}\right)$, for all $x \in \Omega$ and $i \in\{1, \ldots, N\}$ we have

$$
-\frac{1}{\theta} a_{i}\left(x, \partial_{x_{i}} u_{n}\right) \partial_{x_{i}} u_{n} \geq-\frac{P_{+}^{+}}{\theta} A_{i}\left(x, \partial_{x_{i}} u_{n}\right) .
$$

On the other hand, we have by the Lemma 2.1 and Proposition 2.1

$$
\begin{aligned}
\int_{\Omega} \lambda(x)\left|u_{n}\right|^{\alpha(x)} & \leq\left.\left. 2|\lambda|_{\frac{s(x)}{s(x)-\alpha(x)}}|| u_{n}\right|^{\alpha(x)}\right|_{\frac{s(x)}{\alpha(x)}} \\
& \leq 2|\lambda|_{\frac{s(x)}{s(x)-\alpha(x)}}\left|u_{n}\right|_{s(x)}^{\tilde{\alpha}},
\end{aligned}
$$

where $\tilde{\alpha} \in\left[\alpha^{-}, \alpha^{+}\right]$.

Since the embedding $X \hookrightarrow L^{s(x)}(\Omega)$ is compact, then there exists a constant $c_{1}>0$ such that

$$
\left|u_{n}\right|_{s(x)} \leq c_{1}\left\|u_{n}\right\|
$$

Then,

$$
\int_{\Omega} \lambda(x)\left|u_{n}\right|^{\alpha(x)} \leq C|\lambda|_{\frac{s(x)}{s(x)-\alpha(x)}}\left\|u_{n}\right\|^{\tilde{\alpha}},
$$

where $C>0$. From (3.1) and (3.2), we get

$$
1+R+\left\|u_{n}\right\| \geq\left(1-\frac{p_{+}^{+}}{\theta}\right) \sum_{i=1}^{N} \int_{\Omega} A_{i}\left(x, \partial_{x_{i}} u_{n}\right) d x-C_{1}|\lambda|_{\frac{s(x)}{s(x)-\alpha(x)}}\left\|u_{n}\right\|^{\tilde{\alpha}},
$$


where $C_{1}>0$. Again from $\left(A_{2}\right)$ we have

$$
A_{i}\left(x, \partial_{x_{i}} u_{n}\right) \geq \frac{1}{p_{i}(x)}\left|\partial_{x_{i}} u_{n}\right|^{p_{i}(x)} \geq \frac{1}{P_{+}^{+}}\left|\partial_{x_{i}} u_{n}\right|^{p_{i}(x)},
$$

for all $x \in \Omega$ and $i \in\{1, \ldots, N\}$, so

$$
1+R+\left\|u_{n}\right\| \geq\left(\frac{1}{P_{+}^{+}}-\frac{1}{\theta}\right) \sum_{i=1}^{N} \int_{\Omega}\left|\partial_{x_{i}} u_{n}\right|^{p_{i}(x)} d x-C_{1}|\lambda|_{\frac{s(x)}{s(x)-\alpha(x)}}\left\|u_{n}\right\|^{\tilde{\alpha}} .
$$

Using 2. of Lemma 3.1, we get

$$
1+R+\left\|u_{n}\right\| \geq\left(\frac{1}{P_{+}^{+}}-\frac{1}{\theta}\right)\left(\frac{\left\|u_{n}\right\|^{P_{-}^{-}}}{N^{P_{-}^{-}-1}}-N\right)-C_{1}|\lambda|_{\frac{s(x)}{s(x)-\alpha(x)}}\left\|u_{n}\right\|^{\tilde{\alpha}},
$$

and consequently $\left\{u_{n}\right\}$ is bounded because $\theta>P_{+}^{+}$and $P_{-}^{-}>\tilde{\alpha}$. As $X$ is reflexive, then there exists a subsequence still denoted by $\left\{u_{n}\right\}$ which converges weakly to $u_{0}$ in $X$.

Using the fact that $\Phi^{\prime}\left(u_{n}\right) \rightarrow 0$, as $n \rightarrow+\infty$, we can deduce that:

$$
\lim _{n \rightarrow \infty}\left\langle\Phi^{\prime}\left(u_{n}\right), u_{n}-u_{0}\right\rangle=0
$$

more precisely,

$$
\begin{gathered}
\lim _{n \rightarrow \infty} \int_{\Omega}\left[\sum_{i=1}^{N} a_{i}\left(x, \partial_{x_{i}} u_{n}\right)\left(\partial_{x_{i}} u_{n}-\partial_{x_{i}} u_{0}\right)\right. \\
\left.+b(x)\left|u_{n}\right|^{P_{+}^{+}-2} u_{n}\left(u_{n}-u_{0}\right)-\lambda(x) f\left(x, u_{n}\right)\left(u_{n}-u_{0}\right)-\mu(x) g\left(x, u_{n}\right)\left(u_{n}-u_{0}\right)\right] d x=0 .
\end{gathered}
$$

Using Hölder inequality we have

$$
\begin{gathered}
\int_{\Omega} \lambda(x) f\left(x, u_{n}\right)\left(u_{n}-u_{0}\right) \leq\left.\left. 3 c|\lambda|_{\frac{s(x)}{s(x)-\alpha(x)}}|| u\right|^{\alpha(x)-1}\right|_{\frac{s(x)}{\alpha(x)-1}}\left|u_{n}-u_{0}\right|_{s(x)} . \\
\int_{\Omega} \mu(x) g\left(x, u_{n}\right)\left(u_{n}-u_{0}\right) \leq\left.\left. 3 d|\mu|_{\frac{r(x)}{r(x)-\gamma(x)}}|| u\right|^{\gamma(x)-1}\right|_{\frac{r(x)}{\gamma(x)-1}}\left|u_{n}-u_{0}\right|_{r(x)} . \\
\int_{\Omega} b(x)\left|u_{n}\right|^{P_{+}^{+}-2} u_{n}\left(u_{n}-u_{0}\right) \leq\left.\left. 2|b|_{\infty}|| u_{n}\right|^{P_{+}^{+}-1}\right|_{\frac{P_{+}^{+}}{P_{+}^{+}-1}}\left|u_{n}-u_{0}\right|_{P_{+}^{+}} .
\end{gathered}
$$


As $s(x), r(x)$ and $P_{+}^{+}$fulfill Proposition 2.3, thus $\left(u_{n}\right)$ converges strongly to $u_{0}$ in $L^{s(x)}(\Omega), L^{r(x)}(\Omega)$ and $L^{P_{+}^{+}}(\Omega)$. By these facts the relation above reduces to

$$
\lim _{n \rightarrow \infty} \int_{\Omega} \sum_{i=1}^{N} a_{i}\left(x, \partial_{x_{i}} u_{n}\right)\left(\partial_{x_{i}} u_{n}-\partial_{x_{i}} u_{0}\right) d x=0 .
$$

Using Proposition 2.4, we deduce that $\left(u_{n}\right)$ converges strongly to $u_{0}$ in $X$, that is to say that $\Phi$ satisfies Palais-Smale condition.

Since $X$ is a reflexive and separable Banach space, then $X^{*}$ is too. Then there exist (see[11]) $\left\{e_{j}\right\} \subset X$ and $\left\{e_{j}^{*}\right\} \subset X^{*}$ such that

$$
X=\overline{\operatorname{span}\left\{e_{j}: j=1,2 \ldots .\right\}}, X^{*}=\overline{\operatorname{span}\left\{e_{j}^{*}: j=1,2 \ldots .\right\}},
$$

and

$$
\left\langle e_{i}, e_{j}^{*}\right\rangle=\delta_{i j}
$$

Now, we define

$$
X_{j}=\operatorname{span}\left\{e_{j}\right\}, Y_{k}=\bigoplus_{j=1}^{k} X_{j}, Z_{k}=\overline{\bigoplus_{j=k}^{\infty} X_{j}} .
$$

Lemma 3.3. (see[10]) Suppose that $r(x), s(x) \in C_{+}(\bar{\Omega})$ and $r(x), s(x)<$ $P_{-, \infty}$, for all $x \in \bar{\Omega}$. Denote

$$
\beta_{k}=\sup \left\{|u|_{r(x)}:\|u\|=1, u \in Z_{k}\right\}, \theta_{k}=\sup \left\{|u|_{s(x)}:\|u\|=1, u \in Z_{k}\right\} .
$$

Then,

$$
\lim _{k \rightarrow \infty} \beta_{k}=0, \lim _{k \rightarrow \infty} \theta_{k}=0
$$

Lemma 3.4. For every $k \in \mathbf{N}$, there exists $r_{k}>0$ such that

$$
\inf _{u \in Z_{k},\|u\|=r_{k}} \Phi(u) \rightarrow \infty, \text { as } k \rightarrow+\infty .
$$

Proof. By $\left(A_{2}\right),(B),\left(f_{0}\right),\left(g_{0}\right)$, Proposition 2.1, Lemma 2.1 and 2. Lemma 3.1 , for any $u \in Z_{k},\|u\|=r_{k}>1$, when $\mu(x)>0$, a.e. $x \in \Omega$, and $\lambda(x) \in \mathbf{R}$, we have 


$$
\begin{aligned}
\Phi(u) & =\int_{\Omega}\left\{\sum_{i=1}^{N} A_{i}\left(x, \partial_{x_{i}}(u)\right)+\frac{b(x)}{P_{+}^{+}}|u|^{P_{+}^{+}}-\lambda(x) F(x, u)-\mu(x) G(x, u)\right\} d x \\
& \geq \frac{1}{P_{+}^{+}} \sum_{i=1}^{N} \int_{\Omega}\left|\partial_{x_{i}}(u)\right|^{p_{i}(x)} d x+\frac{b_{0}}{P_{+}^{+}} \int_{\Omega}|u|^{P_{+}^{+}} d x-c \int_{\Omega} \frac{|\lambda(x)|}{\alpha(x)}|u|^{\alpha(x)} d x \\
& -d \int_{\Omega} \frac{\mu(x)}{\gamma(x)}|u|^{\gamma(x)} d x \\
& \geq \frac{\|u\|_{-}^{P_{-}^{-}}}{P_{+}^{+} N^{P_{-}^{-}-1}}-C_{1}^{\prime}|\lambda|_{\frac{s(x)}{s(x)-\alpha(x)}}\|u\|^{\tilde{\alpha}}-\frac{2 d}{\gamma^{-}}|\mu|_{\frac{r(x)}{r(x)-\gamma(x)}}|u|_{r(x)}^{\tilde{\gamma}}-\frac{N}{P_{+}^{+}},
\end{aligned}
$$

where $C_{1}^{\prime}=\frac{2 c c_{1}}{\alpha^{-}}$. Since $P_{-}^{-}>\alpha^{+}>\tilde{\alpha}$, there exists $r_{0}$ large enough such that

$$
C_{1}^{\prime}|\lambda|_{\frac{s(x)}{s(x)-\alpha(x)}}\|u\|^{\tilde{\alpha}} \leq \frac{\|u\|^{P_{-}^{-}}}{2 P_{+}^{+} N^{P_{-}^{-}-1}} \text { as } r=\|u\| \geq r_{0} .
$$

If $|u|_{r(x)} \leq 1$ then $|u|_{r(x)}^{r^{+}} \leq|u|_{r(x)}^{\tilde{\gamma}} \leq 1$. But, if $|u|_{r(x)}>1$ using Lemma 3.3, we obtain $|u|_{r(x)}^{\tilde{\gamma}} \leq|u|_{r(x)}^{r^{+}} \leq\left(\beta_{k}\|u\|\right)^{r^{+}}$.

so

$$
\begin{aligned}
& \Phi(u) \geq\left\{\|u\|^{P_{-}^{-}} 2 P_{+}^{+} N^{P_{-}^{-}-1}-\frac{2 d}{\gamma^{-}}|\mu|_{\frac{r(x)}{r(x)-\gamma(x)}}-\frac{N}{P_{+}^{+}} \text {if }|u|_{r(x)} \leq 1,\right. \\
& \frac{\|u\|_{-}^{P_{-}^{-}}}{2 P_{+}^{+} N^{P_{-}^{-}-}}-\frac{2 d}{\gamma^{-}}|\mu|_{\frac{r(x)}{r(x)-\gamma(x)}}\left(\beta_{k}\|u\|\right)^{r^{+}}-\frac{N}{P_{+}^{+}} \text {if }|u|_{r(x)}>1,
\end{aligned}
$$

then

$$
\Phi(u) \geq \frac{\|u\|^{P_{-}^{-}}}{2 P_{+}^{+} N^{P_{-}^{-}-1}}-\frac{2 d}{\gamma^{-}}|\mu|_{\frac{r(x)}{r(x)-\gamma(x)}}\left(\beta_{k}\|u\|\right)^{r^{+}}-C_{12} .
$$

Let's take $r_{k}=\left(\frac{4 d}{\gamma-}|\mu|_{\frac{r(x)}{r(x)-\gamma(x)}} N^{P_{-}^{-}-1} r^{+} \beta_{k}^{r^{+}}\right)^{\frac{1}{P_{-}^{-}-r^{+}}}$. Then,

$$
\Phi(u) \geq \frac{1}{2 N^{P_{-}^{-}-1}}\left(\frac{1}{P_{+}^{+}}-\frac{1}{r^{+}}\right) r_{k}^{P_{-}^{-}}-C_{12},
$$

consequently, $\lim _{k \rightarrow+\infty} \Phi(u)=+\infty$, because $P_{+}^{+}<r^{+}$and $\beta_{k} \rightarrow 0$ when $k \rightarrow+\infty$. 
Lemma 3.5. For every $k \in \mathbf{N}$, there exists $\rho_{k}>r_{k}\left(r_{k}\right.$ given as above) such that

$$
\max _{u \in Y_{k},\|u\|=\rho_{k}} \Phi(u) \leq 0 .
$$

Proof. From $\left(A_{0}\right)$ and $\left(A_{1}\right),\left(f_{0}\right),\left(g_{1}\right)$ and for any $u \in Y_{k}-\{0\}$ with $\|u\|=1$ and $1<\rho_{k}, \mu(x)>0$ a.e. $x \in \Omega$, and $\lambda(x) \in \mathbf{R}$, we have

$$
\begin{aligned}
\Phi\left(\rho_{k} u\right) & =\int_{\Omega}\left\{\sum_{i=1}^{N} A_{i}\left(x, \partial_{x_{i}}\left(\rho_{k} u\right)\right)+\frac{b(x)}{P_{+}^{+}}\left|\rho_{k} u\right|^{P_{+}^{+}}-\lambda(x) F\left(x, \rho_{k} u\right)-\mu(x) G\left(x, \rho_{k} u\right)\right\} d x, \\
& \leq C \sum_{i=1}^{N} \int_{\Omega}\left(\left|\partial_{x_{i}}\left(\rho_{k} u\right)\right|+\frac{\left|\partial_{x_{i}}\left(\rho_{k} u\right)\right|^{p_{i}(x)}}{p_{i}(x)}\right) d x+\frac{\rho_{k}^{P_{+}^{+}}}{P_{+}^{+}} \int_{\Omega} b(x)|u|^{P_{+}^{+}} d x \\
& -\int_{\Omega} \lambda(x) F\left(x, \rho_{k} u\right) d x-\int_{\Omega} \mu(x) G\left(x, \rho_{k} u\right) d x, \\
& \leq C \rho_{k}^{P_{+}^{+}} \sum_{i=1}^{N} \int_{\Omega}\left(\left|\partial_{x_{i}} u\right|+\frac{\left|\partial_{x_{i}} u\right|^{p_{i}(x)}}{P_{-}^{-}}\right) d x+\frac{\rho_{k}^{P_{+}^{+}}}{P_{+}^{+}} \int_{\Omega} b(x)|u|^{P_{+}^{+}} d x \\
& +\frac{c}{\alpha^{-}} \rho_{k}^{\alpha^{+}} \int_{\Omega}|\lambda(x)||u|^{\alpha(x)} d x,-M \rho_{k}^{\theta} \int_{\Omega} \mu(x)|u|^{\theta} d x .
\end{aligned}
$$

We have $Y_{k}$ is a finite dimensional space then all norms are equivalent. Since $\theta>P_{+}^{+}>\alpha^{+}$and $\rho_{k} \rightarrow+\infty$ as $k \rightarrow+\infty$. Hence, for $\rho_{k}$ large enough $\left(\rho_{k}>r_{k}\right), \max _{u \in Y_{k},\|u\|=\rho_{k}} \Phi(u) \leq 0$.

Lemma 3.6. There is $k_{0}$ such that for each $k>k_{0}$, there exists $\rho_{k}>0$ such that $\inf _{u \in Z_{k},\|u\|=\rho_{k}} \Phi(u) \geq 0$.

Proof. From $\left(A_{2}\right),(\mathrm{B}),\left(f_{0}\right),\left(g_{0}\right)$ and Proposition 2.1, Lemma 2.1, and 1. of Lemma 3.1, for any $u \in Z_{k}$, with $\lambda(x)>0$ a.e. $x \in \Omega$, and $\mu(x) \in \mathbf{R}$, one has

$$
\begin{aligned}
\Phi(u) & =\int_{\Omega}\left\{\sum_{i=1}^{N} A_{i}\left(x, \partial_{x_{i}}(u)\right)+\frac{b(x)}{P_{+}^{+}}|u|^{P_{+}^{+}}-\lambda(x) F(x, u)-\mu(x) G(x, u)\right\} d x \\
& \geq \frac{1}{P_{+}^{+}} \sum_{i=1}^{N} \int_{\Omega}\left|\partial_{x_{i}}(u)\right|^{p_{i}(x)} d x+\frac{b_{0}}{P_{+}^{+}} \int_{\Omega}|u|^{P_{+}^{+}} d x-c \int_{\Omega} \frac{\lambda(x)}{\alpha(x)}|u|^{\alpha(x)} d x
\end{aligned}
$$




$$
\begin{aligned}
& -d \int_{\Omega} \frac{|\mu(x)|}{\gamma(x)}|u|^{\gamma(x)} d x, \\
& \geq \frac{\|u\|^{P_{+}^{+}}}{P_{+}^{+} N^{P_{+}^{+}-1}}-\frac{2 c}{\alpha^{-}}|\lambda|_{\frac{s(x)}{s(x)-\alpha(x)}}|u|_{s(x)}^{\tilde{\alpha}}-C_{2}^{\prime}|\mu|_{\frac{r(x)}{r(x)-\gamma(x)}}\|u\|^{\tilde{\gamma}},
\end{aligned}
$$

where $C_{2}^{\prime}=\frac{2 d d_{1}}{\gamma^{-}}$. As $P_{+}^{+}<\gamma^{-} \leq \tilde{\gamma}$, then there exists $\rho_{0}$ small enough such that $C_{2}^{\prime}|\mu|_{\frac{r(x)}{r(x)-\gamma(x)}}\|u\|^{\tilde{\gamma}} \leq \frac{\|u\|_{+}^{P_{+}^{+}}}{2 P_{+}^{+} N^{P_{+}^{+}}}$as $0<\rho=\|u\|<\rho_{0}$. So using lemma 3.3 , we have

$$
\begin{gathered}
\Phi(u) \geq \\
\left\{\|u\|^{P_{+}^{+}} 2 P_{+}^{+} N^{P_{+}^{+}-1}-\frac{2 c}{\alpha^{-}}|\lambda|_{\frac{s(x)}{s(x)-\alpha(x)}} \text { if }|u|_{s(x)} \leq 1,\right. \\
\frac{\|u\|_{+}^{P_{+}^{+}}}{2 P_{+}^{+} N^{P_{+}^{+}-1}}-\frac{2 c}{\alpha^{-}}|\lambda|_{\frac{s(x)}{s(x)-\alpha(x)}}\left(\theta_{k}\|u\|\right)^{\alpha^{+}} \text {if }|u|_{s(x)}>1 .
\end{gathered}
$$

Let's take $\rho_{k}=\left(\frac{4 c}{\alpha^{-}} P_{+}^{+} N^{P_{+}^{+}-1}|\lambda|_{\frac{s(x)}{s(x)-\alpha(x)}} \theta_{k}^{\alpha^{+}}\right)^{\frac{1}{P_{+}^{+}-\alpha^{+}}}$. As $P_{+}^{+}>P_{-}^{-}>$ $\alpha^{+}$, then $\theta_{k} \rightarrow 0$ means $\rho_{k} \rightarrow 0$ as $k$ goes to $+\infty$. It follows from the inequality above that

$$
\Phi(u) \geq \frac{\rho_{k}^{P_{+}^{+}}}{2 P_{+}^{+} N^{P_{+}^{+}-1}}-\frac{\rho_{k}^{P_{+}^{+}}}{2 P_{+}^{+} N^{P_{+}^{+}-1}}=0 .
$$

Finally, we deduce that for $u \in Z_{k}$ with $\|u\|=\rho_{k}, \Phi(u) \geq 0$.

Lemma 3.7. for each $k>k_{0}$, there exists $r_{k}<\rho_{k}$ ( $\rho_{k}$ given by Lemma 3.6) such that $\max _{u \in Y_{k},\|u\|=r_{k}} \Phi(u)<0$, as $k \rightarrow+\infty$.

Proof. From $\left(A_{0}\right),\left(A_{1}\right),\left(f_{0}\right),\left(f_{1}\right),\left(g_{0}\right)$, and Proposition 2.1, for any $v \in$ $Y_{k}$ with $\|v\|=1$ and $0<t<\rho_{k}<1$, with $\lambda(x)>0$ a.e. $x \in \Omega$, and $\mu(x) \in \mathbf{R}$, we have

$$
\Phi(t v)=\int_{\Omega}\left\{\sum_{i=1}^{N} A_{i}\left(x, \partial_{x_{i}}(t v)\right)+\frac{b(x)}{P_{+}^{+}}|t v|^{P_{+}^{+}}-\lambda(x) F(x, t v)-\mu(x) G(x, t v)\right\} d x
$$




$$
\begin{aligned}
& \leq C \sum_{i=1}^{N} \int_{\Omega}\left(\left.\left|\partial_{x_{i}}\left(t v \mid+\frac{\left|\partial_{x_{i}}(t v)\right|^{p_{i}(x)}}{p_{i}(x)}\right) d x+\frac{t^{P_{+}^{+}}}{P_{+}^{+}} \int_{\Omega} b(x)\right| v\right|^{P_{+}^{+}} d x\right. \\
& -\int_{\Omega} \lambda(x) F(x, t v) d x-\int_{\Omega} \mu(x) G(x, t v) d x \\
& \leq C t \sum_{i=1}^{N} \int_{\Omega}\left(\left|\partial_{x_{i}} v\right|+\frac{\left|\partial_{x_{i}} v\right|^{p_{i}(x)}}{P_{-}^{-}}\right) d x+\frac{t^{P_{+}^{+}}}{P_{+}^{+}} \int_{\Omega} b(x)|v|^{P_{+}^{+}} d x \\
& -t^{\alpha_{0}^{+}} \int_{\Omega} \lambda(x) h_{0}(x)|v|^{\alpha_{0}(x)} d x+\frac{d}{\gamma^{-}} t^{\gamma^{-}} \int_{\Omega}|\mu(x)||v|^{\gamma(x)} d x .
\end{aligned}
$$

Since $\gamma^{-}>1>\alpha_{0}^{+}$and $\operatorname{dim} Y_{k}=k$, then there exists $0<r_{k}<\rho_{k}$ such that $\Phi(u)<0$ if $\|u\|=r_{k}$. Hence $b_{k}=\max _{u \in Y_{k},\|u\|=r_{k}} \Phi(u)<0$.

Lemma 3.8. For each $k>k_{0}$, and $\rho_{k}>0$ given by Lemma 3.6, one has

$$
\inf _{u \in Z_{k},\|u\| \leq \rho_{k}} \Phi(u) \rightarrow 0, \text { as } k \rightarrow+\infty .
$$

Proof. From a definition of $Y_{k}$ and $Z_{k}$, we have $Y_{k} \cap Z_{k} \neq$ and $r_{k}<\rho_{k}$, then

$$
d_{k}=\inf _{u \in Z_{k},\|u\| \leq \rho_{k}} \Phi(u) \leq b_{k}=\max _{u \in Y_{k},\|u\|=r_{k}} \Phi(u)<0 .
$$

From ( 3.3), for $u \in Z_{k},\|u\| \leq \rho_{k}$ small enough we have

$$
\begin{aligned}
\Phi(u) & \geq \frac{\|u\|_{+}^{P_{+}^{+}}}{2 P_{+}^{+} N^{P_{+}^{+}-1}}-\frac{2 c}{\alpha^{-}}|\lambda|_{\frac{s(x)}{s(x)-\alpha(x)}}\left(\theta_{k}\|u\|\right)^{\alpha^{+}}, \\
& \geq-\frac{2 c}{\alpha^{-}}|\lambda|_{\frac{s(x)}{s(x)-\alpha(x)}} \theta_{k}^{\alpha^{+}}\|u\|^{\alpha^{+}} .
\end{aligned}
$$

We deduce that $d_{k} \rightarrow 0$, because $\theta_{k} \rightarrow 0$ and $\rho_{k} \rightarrow 0$ as $k \rightarrow+\infty$.

Definition 3.1. The functional $\Phi$ satisfies the $(P S)_{c}^{*}$ condition (with respect to $\left.\left(Y_{n}\right)\right)$, if any sequence $\left(u_{n_{j}}\right) \subset X$ such that $n_{j} \rightarrow+\infty, u_{n_{j}} \in$ $Y_{n_{j}}, \Phi\left(u_{n_{j}}\right) \rightarrow c$ and $\left(\left.\Phi\right|_{Y_{n_{j}}}\right)^{\prime}\left(u_{n_{j}}\right) \rightarrow 0$, has a subsequence converging to a critical point of $\Phi$.

Lemma 3.9. The functional $\Phi$ satisfies the $(P C)_{c}^{*}$ for every $c \in\left[d_{k_{0}}, 0[\right.$. 
Proof. Assume that $\left(u_{n_{j}}\right) \subset X$ such that $n_{j} \rightarrow+\infty, u_{n_{j}} \in Y_{n_{j}}, \Phi\left(u_{n_{j}}\right) \rightarrow$ $c$ and $\left(\left.\Phi\right|_{Y_{n_{j}}}\right)^{\prime}\left(u_{n_{j}}\right) \rightarrow 0$. Suppose that $\left\|u_{n_{j}}\right\|>1$. If $\mu(x)>0$ a.e. $x \in \Omega$, for $n_{j}$ large enough, we have

$$
\begin{aligned}
1+R_{1}+\left\|u_{n_{j}}\right\| & \geq \Phi\left(u_{n_{j}}\right)-\frac{1}{\theta}\left\langle\Phi^{\prime}\left(u_{n_{j}}\right), u_{n_{j}}\right\rangle, \\
& \geq\left(\frac{1}{P_{+}^{+}}-\frac{1}{\theta}\right)\left(\frac{\left\|u_{n_{j}}\right\|^{-}}{N^{P_{-}^{-}-1}}-N\right)-M_{1}|\lambda|_{\frac{s(x)}{s(x)-\alpha(x)}}\left\|u_{n_{j}}\right\|^{\tilde{\alpha}} .
\end{aligned}
$$

Since $\theta>P_{+}^{+}$and $P_{-}^{-}>\tilde{\alpha},\left\{u_{n_{j}}\right\}$ is bounded in $X$.

For a subsequence $\left\{u_{n_{j}}\right\}$ we have $u_{n_{j}} \rightarrow u \in X$. As $X=\overline{\bigcup_{n_{j}} Y_{n_{j}}}$, we can take $v_{n_{j}} \in Y_{n_{j}}$ such that $v_{n_{j}} \rightarrow u$. Then

$$
\begin{aligned}
\lim _{n_{j} \rightarrow+\infty}\left\langle\Phi^{\prime}\left(u_{n_{j}}\right), u_{n_{j}}-u\right\rangle & =\lim _{n_{j} \rightarrow+\infty}\left\langle\Phi^{\prime}\left(u_{n_{j}}\right), u_{n_{j}}-v_{n_{j}}\right\rangle \\
& +\lim _{n_{j} \rightarrow+\infty}\left\langle\Phi^{\prime}\left(u_{n_{j}}\right), v_{n_{j}}-u\right\rangle, \\
& =\lim _{n_{j} \rightarrow+\infty}\left\langle\left(\left.\Phi\right|_{Y_{n_{j}}}\right)^{\prime}\left(u_{n_{j}}\right), u_{n_{j}}-v_{n_{j}}\right\rangle, \\
& =0 .
\end{aligned}
$$

We follow the similar procedure in the proof of $(P S)$ condition in the Lemma 3.2. We conclude that $u_{n_{j}} \rightarrow u$, therefore we have $\Phi^{\prime}\left(u_{n_{j}}\right) \rightarrow \Phi^{\prime}(u)$. Let's show that $\Phi^{\prime}(u)=0$. Let us take $\varphi_{k} \in Y_{k}$, notice that if $n_{j} \geq k$ we have

$$
\begin{aligned}
\left\langle\Phi^{\prime}(u), \varphi_{k}\right\rangle & =\left\langle\Phi^{\prime}(u)-\Phi^{\prime}\left(u_{n_{j}}\right), \varphi_{k}\right\rangle+\left\langle\Phi^{\prime}\left(u_{n_{j}}\right), \varphi_{k}\right\rangle, \\
& =\left\langle\Phi^{\prime}(u)-\Phi^{\prime}\left(u_{n_{j}}\right), \varphi_{k}\right\rangle+\left\langle\left(\left.\Phi\right|_{Y_{n_{j}}}\right)^{\prime}\left(u_{n_{j}}\right), \varphi_{k}\right\rangle .
\end{aligned}
$$

Then, when $n_{j} \rightarrow+\infty$, we obtain

$$
\left\langle\Phi^{\prime}(u), \varphi_{k}\right\rangle=0 \forall \varphi_{k} \in Y_{k},
$$

so $\Phi^{\prime}(u)=0$. Finally, $\Phi$ satisfies $(P S)_{c}^{*}$ condition for every $c \in \mathbf{R}$.

Proof theorem 1.1. 
By using Fountain theorem ( see[20], [4]), Lemma 3.2, Lemma 3.4 and Lemma 3.5, and as $\Phi$ is even the proof of 1 . Theorem 1.1 is finished.

Now, we apply the dual fountain theorem ( see[20], [4]) in order to prove 2. and 3. of Theorem 1.1, first by using the Lemma 3.6, Lemma 3.7, Lemma 3.8 and Lemma 3.9, the conditions of dual fountain theorem are fulfilled. As $\Phi$ is even, this achieved the proof of 2 . of Theorem 1.1.

\section{Proof of 3. of theorem 1.1.}

In order to prove 3 . of theorem 1.1, we apply the dual fountain theorem, and we use the condition $\left(g_{1}^{\prime}\right)$, because the only problem here is to demonstrate that every $(P S)^{*}$ sequence is bounded. In the fact, assume that $\left(u_{n_{j}}\right) \subset X$ such that $n_{j} \rightarrow+\infty, u_{n_{j}} \in Y_{n_{j}}, \Phi\left(u_{n_{j}}\right) \rightarrow c$ and $\left(\left.\Phi\right|_{Y_{n_{j}}}\right)^{\prime}\left(u_{n_{j}}\right) \rightarrow$ 0 . Suppose that $\left\|u_{n_{j}}\right\|>1$. For the case $\lambda(x)>0$ and $\mu(x)>0$ a.e. $x \in \Omega$, the proof is the same as above. Then we will treat only the case when $\lambda(x)>0$ and $\mu(x)<0$ a.e. $x \in \Omega$.

For $n_{j}$ large enough, we have

$$
\begin{aligned}
& 1+R_{2}+\left\|u_{n_{j}}\right\| \geq \Phi\left(u_{n_{j}}\right)-\frac{1}{\beta}\left\langle\Phi^{\prime}\left(u_{n_{j}}\right), u_{n_{j}}\right\rangle \\
& \geq \int_{\Omega}\left\{\sum_{i=1}^{N} A_{i}\left(x, \partial_{x_{i}} u_{n_{j}}\right)+\frac{b(x)}{P_{+}^{+}}\left|u_{n_{j}}\right|^{P_{+}^{+}}-\lambda(x) F\left(x, u_{n_{j}}\right)\right. \\
& \text { - } \left.\mu(x) G\left(x, u_{n_{j}}\right)\right\} d x \\
& -\frac{1}{\beta} \int_{\Omega}\left\{\sum_{i=1}^{N} a_{i}\left(x, \partial_{x_{i}} u_{n_{j}}\right) \partial_{x_{i}} u_{n_{j}}+b(x)\left|u_{n_{j}}\right|^{P_{+}^{+}}\right. \\
& \left.-\lambda(x) f\left(x, u_{n_{j}}\right) u_{n_{j}}-\mu(x) g\left(x, u_{n_{j}}\right) u_{n_{j}}\right\} d x \\
& \geq \int_{\Omega}\left\{\sum_{i=1}^{N} A_{i}\left(x, \partial_{x_{i}} u_{n_{j}}\right)-\frac{1}{\beta} a_{i}\left(x, \partial_{x_{i}} u_{n_{j}}\right) \partial_{x_{i}} u_{n_{j}}\right\} d x \\
& +\left(\frac{1}{P_{+}^{+}}-\frac{1}{\beta}\right) \int_{\Omega} b(x)\left|u_{n_{j}}\right|^{P_{+}^{+}} d x \\
& +\int_{\Omega} \mu(x)\left(\frac{1}{\beta} g\left(x, u_{n_{j}}\right) u_{n_{j}}-G\left(x, u_{n_{j}}\right)\right) d x \\
& +\int_{\Omega} \lambda(x)\left(\frac{1}{\beta} f\left(x, u_{n_{j}}\right) u_{n_{j}}-F\left(x, u_{n_{j}}\right)\right) d x
\end{aligned}
$$




$$
\begin{aligned}
& \geq \int_{\Omega}\left\{\sum_{i=1}^{N} A_{i}\left(x, \partial_{x_{i}} u_{n_{j}}\right)-\frac{1}{\beta} a_{i}\left(x, \partial_{x_{i}} u_{n_{j}}\right) \partial_{x_{i}} u_{n_{j}}\right\} d x \\
& -c\left(\frac{1}{\beta}+\frac{1}{\alpha^{-}}\right) \int_{\Omega}|\lambda(x)|\left|u_{n_{j}}\right|^{\alpha(x)} .
\end{aligned}
$$

Then,

$$
\begin{aligned}
1+R_{2}+\left\|u_{n_{j}}\right\| & \geq \Phi\left(u_{n_{j}}\right)-\frac{1}{\beta}\left\langle\Phi^{\prime}\left(u_{n_{j}}\right), u_{n_{j}}\right\rangle, \\
& \geq\left(\frac{1}{P_{+}^{+}}-\frac{1}{\beta}\right)\left(\frac{\left\|u_{n_{j}}\right\|^{P_{-}^{-}}}{N^{P_{-}^{-}-1}}-N\right)-M_{2}|\lambda|_{\frac{s(x)}{s(x)-\alpha(x)}}\left\|u_{n_{j}}\right\|^{\tilde{\alpha}} .
\end{aligned}
$$

Since $\beta>P_{+}^{+}$and $P_{-}^{-}>\tilde{\alpha},\left\{u_{n_{j}}\right\}$ is bounded in $X$. This achieve the proof.

\section{References}

[1] G. A. Afrouzi, M. Mirzapour, and V. D. Rădulescu, "Qualitative Properties of Anisotropic Elliptic Schrödinger Equations", Advanced Nonlinear Studies, vol. 14, pp. 719-736, 2014.

[2] C. O. Alves, and J. L. P. Barreiro, "Existence and multiplicity of solutions for a $\mathrm{p}(\mathrm{x})$ - Laplacian equation with critical growth", Journal of Mathematical Analysis and Applications, vol. 403, pp. 143-154, 2013.

[3] A. Ambrosetti, and P. H. Rabinowitz, "Dual variational methods in critical points theory and applications", Journal of Functional Analysis, vol. 14, pp. 349-381, 1973.

[4] T. Bartsch, "Infinitely many solutions of a symmetric Dirichlet problema”, Nonlinear Analysis, vol. 20, pp. 1205-1216, 1993.

[5] M. M. Boureanu, "Infinitely many solution for a class of degenerate anisotropic elliptic problems with variable exponent", Taiwanese journal of mathematics, vol. 15, pp. 2291-2310, 2011.

[6] M. M. Boureanu, P. Pucci, and V. D. Rădulescu, "Multiplicity of solutions for a class of anisotropic elliptic equations with variable exponent", Complex Variables and Elliptic Equations, vol. 56, pp. 755-767, 2011. 
[7] H. Brezis, Analyse Fonctionnelle. Théorie, Méthodes et Applications. París: Masson, 1992.

[8] D. E. Edmunds, J. Rákosník, "Sobolev embedding with variable exponent", Studia Mathematica, vol. 143, pp. 267-293, 2000.

[9] X. L. Fan, "Anisotropic variable exponent Sobolev spaces and p (x) -Laplacian equations", Complex Variables and Elliptic Equations, vol. 56, no.7-9, pp. 623-642, 2011.

[10] X. L. Fan, "Solutions for $\mathrm{p}(\mathrm{x})$-Laplacian Dirichlet problems with singular coefficients", Journal of Mathematical Analysis and Applications, vol. 312, pp. 464-477, 2005.

[11] X. L. Fan, and X. Y. Han, "Existence and multiplicity of solutions for $\mathrm{p}(\mathrm{x})$ - Laplacian equations in $\mathbf{R}^{\mathrm{N}}$ ", Nonlinear analysis, vol. 59, pp. 173-188, 2004.

[12] X. L. Fan, J. S. Shen, and D. Zhao, "Sobolev embedding theorems for spaces $\mathrm{W}^{\mathrm{k}, \mathrm{p}(\mathrm{x})}$ ", Journal of Mathematical Analysis and Applications, vol. 262, pp. 749-760, 2001.

[13] X. L. Fan and D. Zhao, "On the spaces $\mathrm{L}^{\mathrm{p}(\mathrm{x})}$ and $\mathrm{W}^{\mathrm{m}} \mathrm{p}(\mathrm{x})$ ", Journal of Mathematical Analysis and Applications, vol. 263, pp. 424-446, 2001.

[14] B. Kone, S. Ouaro, and S. Traore, "Weak solutions for anisotropic nonlinear elliptic equations with variable exponents", Electronic Journal of Differential Equations, vol. 2009, no. 144.pp. 1-11, 2009.

[15] M. Mihǎilescu and G. Moroşanu, "Existence and multiplicity of solutions for an anisotropic elliptic problem involving variable exponent growth conditions", Applicable Analysis, vol. 89, no. 2, pp. 257-271, 2010 .

[16] M. Mihăilescu, P. Pucci, and V. Rǎdulescu, "Eigenvalue problems for anisotropic quasilinear elliptic equations with variable exponent", Journal of Mathematical Analysis and Applications, vol. 340, pp. 687-698, 2008.

[17] T. G. Myers, "Films with high surface tensión”, SIAM Review, vol. 40, no. 3, pp. 441-462, 1998.

[18] H. Nguyen, and K. Schmitt, "Nonlinear elliptic Dirichlet and no-flux boundary value problems", Annals of the University of Bucharest (Mathematical Series), vol. 61, no. 3, pp. 201-217, 2012.

[19] M. Rŭžicka, Electrorheological Fluids: Modeling and Mathematical Theory. Berlin: Springer, 2000. 
[20] M. Willem, Minimax Theorems. Boston: Birkhauser, 1996.

[21] J. F. Zhao, Structure Theory of Banach Spaces. Wuhan: Wuhan University Press, 1991.

[22] L. Zhao, P. Zhao, and X. Xie, "Existence and multiplicity of solutions for divergence type elliptic equations", Electronic Journal of Differential Equations, no. 43, pp. 1-9, 2011.

[23] V. V. Zhikov, S. M. Kozlov, and O. A. Oleinik, Homogenization of Differential Operators and Integral Functionals. Berlin: Springer, 1994.

\author{
Abdelrachid El Amrouss \\ University Mohamed I, \\ Faculty of sciences, \\ Department of Mathematics, \\ Oujda, \\ Morocco \\ e-mail: elamrouss@hotmail.com \\ and
}

\author{
Ali El Mahraoui \\ University Mohamed I, \\ Faculty of sciences, \\ Department of Mathematics, \\ Oujda, \\ Morocco \\ e-mail: alielmahra@gmail.com
}

